Check for updates

Cite this: RSC Adv., 2019, 9, 10264

\title{
Extracellular histones are clinically associated with primary graft dysfunction in human liver transplantation
}

Xiuhui $\mathrm{Li}, \uparrow^{\mathrm{a}}$ Chunyan Gou, $\uparrow^{\mathrm{a}}$ Yanhua Pang, $\hat{\dagger}^{\mathrm{b}}$ Yakun Wang, ${ }^{\mathrm{c}}$ Yan $\mathrm{Liu}^{\mathrm{a}}$ and Tao Wen (DD *c

Extracellular histones have been involved in numerous inflammatory conditions such as ischemia/ reperfusion (I/R) injury, trauma, and infection. There is growing evidence of I/R injury associated with primary graft dysfunction (PGD) following organ transplantation. Here we investigated whether extracellular histones are clinically involved with PGD in human liver transplantation. In total 58 patients undergoing liver transplantation were studied. We collected blood samples from the recipients before and serially after transplantation (24 h, 72 h). We measured extracellular histones, myeloperoxidase (MPO), S100A8/A9, and multiple inflammatory cytokines. Additionally, we exposed human L02 hepatocytes or U937 monocytic cells to the recipient's sera overnight, and assessed cellular viability and cytokine production respectively. Lastly, we assessed the effect of histonetargeted interventions by administration of heparin or an anti-histone antibody. It showed that extracellular histones increased immediately after transplantation, peaked within 24 hours and remained at high levels up to 72 hours (all $p<0.01$ ). Notably, extracellular histone levels were significantly higher in recipients with PGD $(n=9)$ than recipients without PGD $(n=49, p=0.004)$. Extracellular histones correlated positively with MPO, S100A8/A9 and most detected cytokines. Ex vivo analysis demonstrated that the patients' sera after graft markedly induced L02 cell death and caused profound cytokine production in cultured $U 937$ cells, which could be abrogated by heparin or an antihistone antibody. Collectively, extracellular histones were increased significantly after liver transplantation, which may contribute to the occurrence of PGD through direct cytotoxicity and enhancement of systemic inflammation. Targeting extracellular histones may provide a promising approach for preventing PGD or other complications in clinical practice.

Received 17th January 2019 Accepted 28th March 2019

DOI: $10.1039 / c 9 r a 00425 d$

rsc.li/rsc-advances related factors such as age, nutritional status, hemodynamic stability during harvesting, and transplant-related factors such as graft preservation, ischemia/reperfusion (I/R) injury have been proposed as common risk factors for PGD. ${ }^{6,7}$ Of these, I/R injury occurs unavoidably during the course of liver transplantation and is widely accepted as a major culprit of graft dysfunction, which may lead to organ injury via cytotoxicity and inflammation. ${ }^{6,8}$

Liver I/R injury is a complex process involving many types of cells and mediators through various pathways. ${ }^{6,8}$ Recently, extracellular histones have been characterized as key mediators of $\mathrm{I} / \mathrm{R}$ injuries in many organs including the liver, kidney, heart, and brain. ${ }^{9-11}$ Extracellular histones increase remarkably after I/ $\mathrm{R}$ injury, which are either released passively by stressed or injured cells, or are actively secreted by innate immune cells (e.g. neutrophils, monocytes/macrophages). ${ }^{12,13}$ Extracellular histones not only correlate with disease severity and poor outcomes, which can reflect ongoing cellular damage or the inflammatory states, but also serve as a therapeutic target for I/ $\mathrm{R}$ injury. ${ }^{11,14}$ Targeting extracellular histones attenuates the I/R injury of many organs. ${ }^{14,15}$ The mechanisms of histone- 
mediated injury are yet to be fully understood. Available evidence suggests that extracellular histones are cytotoxic to endothelial cells, enhance platelet aggregation and coagulation activation, as well as promote inflammatory responses through TLRs or other inflammatory pathways., ${ }^{\mathbf{9 6 , 1 7}}$ Histone-targeted therapy appears to be protective in various inflammatory injuries including $\mathrm{I} / \mathrm{R}$ injury. ${ }^{\mathbf{1 1}}$

Considering that $\mathrm{I} / \mathrm{R}$ injury is a most important risk factor for PGD in human liver transplantation, we reasoned whether extracellular histones, which are critical mediators for $\mathrm{I} / \mathrm{R}$ injury, may play a pathological and targetable role during this process. The aims of the present study were: (1) to measure extracellular histones in patients who received liver transplantation, (2) to investigate the relationship between extracellular histones and other markers of inflammation (e.g. MPO, S100A8/9, and multiple cytokines), and (3) to examine the effect of extracellular histones on cell cytotoxicity and immune cell activation via ex vivo investigations.

\section{Patients and methods}

\subsection{Patients}

This retrospective study was approved by the Ethics Committee of Beijing Youan Hospital, Capital Medical University (Beijing, P. R. China), which followed the recommendations of the Declaration of Helsinki for biomedical research involving human subjects. Written informed consent was obtained from all patients or their nominated next of kin. Totally 58 patients who underwent liver transplantation between December 2015 and May 2017 were included in this study. Indications for liver transplantation were presented in Table 1. The PGD was evaluated based on the following parameters: aminotransferase activity, bile production, and coagulopathy within 72 hours after transplantation., ${ }^{4,18}$

\subsection{Sample collection}

The peripheral blood samples were collected from these recipients before and serially after transplantation. Totally 3 time points were defined: before graft (T0), within 24 hours after graft (T1), and 72 hours after graft (T2). Sera samples were

Table 1 Baseline characteristics in patients undergoing liver transplantation $^{a}$

\begin{tabular}{ll}
\hline Characteristics & Values \\
\hline Age (years) & $52.0(46.0-61.0)$ \\
Gender (male/female) & $39 / 19$ \\
Etiology of liver disease & \\
Liver cirrhosis (\%) & $22(37.9 \%)$ \\
Hepatocellular cancer (\%) & $20(34.5 \%)$ \\
Acute liver failure (\%) & $9(15.5 \%)$ \\
Others (\%) & $7(12.1 \%)$ \\
MELD score & $17($ range, 9-28)
\end{tabular}

${ }^{a}$ Data are expressed as median (interquartile range) or number of patients (\%). MELD $=$ model for end-stage liver disease. separated, aliquoted and stored at $-80{ }^{\circ} \mathrm{C}$. Baseline characteristics of these recipients were recorded electronically.

\subsection{Measurement of extracellular histones}

We measured extracellular histone levels in the sera of patients using a commercially available sandwich enzyme-linked immunosorbent assay (Cell Death Detection ELISA kit; Roche Applied Science, Germany). ${ }^{19}$ Purified calf thymus histones were used to generate standard curves.

\subsection{Measurement of MPO, S100A8/9 and multiple cytokines}

It is known that the process of $\mathrm{I} / \mathrm{R}$ recruits neutrophils and activated macrophages that exaggerate organ inflammation, tissue injury and malfunction. ${ }^{6,20}$ MPO activity is an index of neutrophil, monocyte/macrophage activation, whereas S100A8/ A9, which is abundantly stored in neutrophil cytoplasm, is another classical marker for neutrophil activation. ${ }^{\mathbf{2 1 , 2 2}}$ Therefore, we assayed both MPO and S100A8/A9 levels to assess activation of innate immune cells particularly neutrophils, in the sera of patients undergoing liver transplantation using commercially available kits.

We also measured a panel of multiple cytokines (IFN- $\gamma$, MCP-1, IL-1 $\beta$, IL-2, IL-4, IL-5, IL-6, IL-9, IL-10, IL-12p70, IL-13, IL-17A, IL-18, IL-21, IL-23, IL-27, and TNF- $\alpha$ ) using the ProcartaPlex ${ }^{\mathrm{TM}}$ Multiplex Immunoassay from Affimetrix eBioscience (San Diego, CA, USA).

\subsection{Ex vivo experiments}

We obtained the human hepatocyte cell line L02 and human monocyte cell line (U937) from American Type Culture Collection (ATCC), and cultured the cells in DMEM (Dulbecco's Modified Eagle's Medium, Sigma-Aldrich, USA) supplemented with $10 \%$ fetal bovine serum (HyClone, UT, USA), 2 mM glutamine, and $100 \mathrm{U}$ per $\mathrm{ml}$ penicillin per streptomycin (SigmaAldrich, USA) in a $5 \% \mathrm{CO}_{2}$ humidified atmosphere at $37{ }^{\circ} \mathrm{C}$. After the cells grew to 80-90\% confluence, they were incubated overnight with $50 \%$ of the sera samples, which were pooled from these recipients before (T0) or after graft (T1) respectively. For interventional studies, anti-histone $\mathrm{H} 4$ antibody $(20 \mu \mathrm{g}$ $\mathrm{ml}^{-1}$ ) or heparin (200 $\mathrm{U} \mathrm{ml}^{-1}$, Sigma-Aldrich, USA) was administered to the cultured cells. The mouse anti-histone $\mathrm{H} 4$ $\mathrm{mAb}$ was prepared following the previous protocol involving autoimmune mice. ${ }^{29,30}$

L02 cell integrity was assessed using the Cell Counting Kit-8 (CCK-8, Sigma-Aldrich, USA), according to the manufacturer's instructions. Briefly, after incubation overnight with the sera, the cells were washed with PBS twice and incubated with $10 \mathrm{ml}$ of CCK-8 solution for 1 hour. The absorbance was measured at $450 \mathrm{~nm}$ using a spectrophotometer.

As for measurement of the stimulatory effects, after U937 cells being treated overnight with the sera, the cell culture supernatants were harvested and analyzed for IL-1 $\beta$, IL-6, IL-10, IL-18, IFN- $\gamma$ and TNF- $\alpha$ levels, using the ProcartaPlex ${ }^{\text {TM }}$ Multiplex Immunoassay from affimetrix eBioscience (San Diego, CA, USA). 


\subsection{Statistical analysis}

For human data, values were expressed as medians and interquartile ranges or percentages unless otherwise stated. For cell culture data, values were expressed as mean orstandard deviation (SD). Data were analyzed using unpaired Student's $t$ test or Mann-Whitney test (for two groups), oneway analysis of variance (ANOVA) followed by Turkey posttests (for more than two groups). Correlations between variables were assessed using Spearman's rank correlation or Pearson correlation analysis. Results were considered statistically significant when $p<0.05$. All statistical analyses were calculated using GraphPad Prism v6 (GraphPad Software, Inc., San Diego, CA).

\section{Results}

\subsection{Patient characteristics}

The baseline characteristics of the recipients were listed in Table 1. A total of 58 adult liver transplantations were performed. Donor livers were obtained from deceased cardiac failure patients. Main reasons for transplantation were liver cirrhosis $(n=22,37.9 \%)$, hepatocellular cancer $(n=20$, $34.5 \%)$, acute liver failure $(n=9,15.5 \%)$ and others $(n=7$, $12.1 \%)$.

\subsection{Histone levels increased greatly after liver transplantation}

It showed that extracellular histone levels in patients after liver transplantation were 5-6 times greater than the levels measured before transplantation. Overall extracellular histones peaked within 24 hours after graft (T1) and tended to decrease within 72 hours (T2), but were still significantly higher than the levels before graft (T0) (Fig. 1A).

We assessed whether these recipients developed PGD, which was defined as graft insufficiency with possible early graft loss, without technical, anatomical, immunological or hepatitisrelated issues. ${ }^{\mathbf{1 , 4}}$ According to an early evaluation (72 h post- transplantation), the recipients were divided into those with PGD $(n=9)$ and those without PGD $(n=49)$. Notably, extracellular histone levels in patients with PGD (median value, 20.99 $\mu \mathrm{g} \mathrm{ml}^{-1}$; range, 18.95-26.54) were significantly higher than in patients without PGD (median value, $16.43 \mu \mathrm{g} \mathrm{ml}{ }^{-1}$; range, 14.29-18.07; $p=0.004$ ) (Fig. 1B).

\subsection{Extracellular histone levels correlated with immune cell activation and systemic inflammation}

It has been reported that high levels of extracellular histones are closely associated with innate immune cell activation and pronounced systemic inflammation. ${ }^{23-25}$ We chose to assay MPO activity and S100A8/A9 levels, which reflect activation of immune cells particularly neutrophils, during the course of liver transplantation. It showed that MPO and S100A8/A9 levels were both elevated significantly at $\mathrm{T} 1$ ( $24 \mathrm{~h}$ post-transplantation), as compared with T0 (before transplantation). MPO and S100A8/ A9 levels exhibited a decreasing trend within 72 hours posttransplantation, but were still higher than the levels at T0 (Fig. 2). There was also a positive correlation between extracellular histones and MPO $(r=0.5262, p=0.003)$ and S100A8/A9 $(r$ $=0.4738, p=0.015)$.

In addition, we evaluated the degree of systemic inflammation in patients after liver transplantation by measuring a panel of cytokines. We found that a total of 10 cytokines was markedly increased in recipients at T1 (24 hour after liver transplantation) as compared with their levels before graft (Fig. 3). At T2 (72 hours after graft), several cytokines (IFN- $\gamma$, IL-1 $\beta$, IL-6, IL-10, IL-18, and TNF- $\alpha$ ) showed a decreasing trend but were still higher than at T0, whereas some cytokines remained at the elevated levels (IL-2, IL-12p70, IL-17A). A line correlation analysis demonstrated that extracellular histone levels were significantly related to most detected cytokines (Table 2). Collectively, these findings suggested that the elevated extracellular histones may account for immune cell activation and the enhanced systemic inflammation after liver transplantation.
A

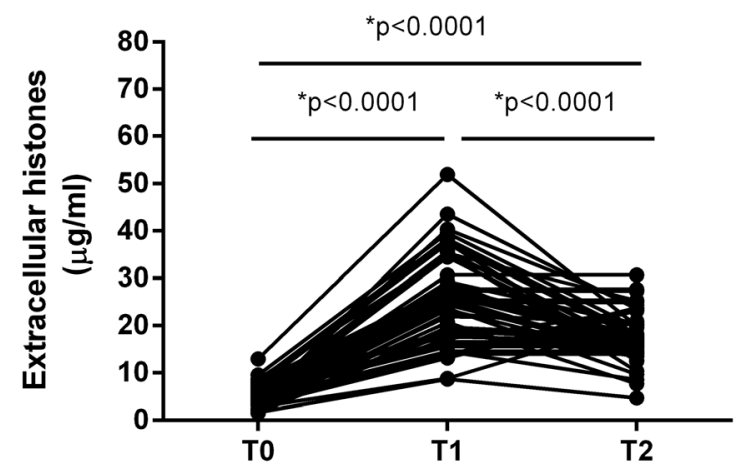

$\mathbf{B}$

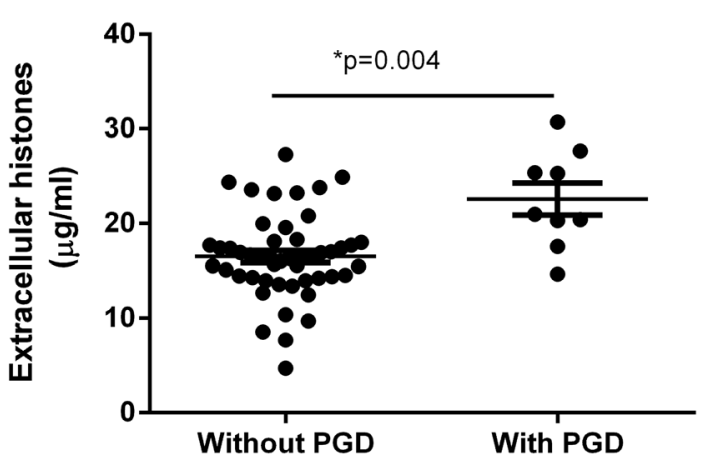

Fig. 1 Extracellular histones were significantly elevated in the sera of patients undergoing liver transplantation. (A) Sequential extracellular histone levels in patients undergoing liver transplantation at T0 (before graft), T1 (24 hours after graft) and T2 (within 72 hours after graft). (B) Median extracellular histones (T2) were significantly higher in patients with PGD than those in patient without PGD $(p=0.004)$. Variables were expressed as median (interquartile range). 
A

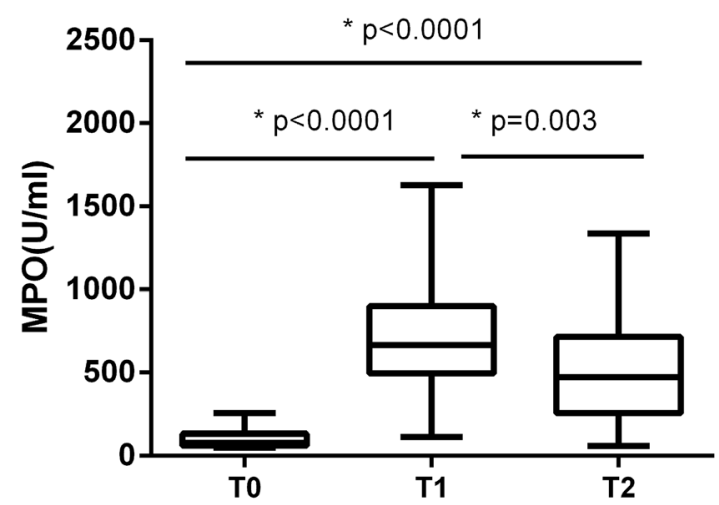

B

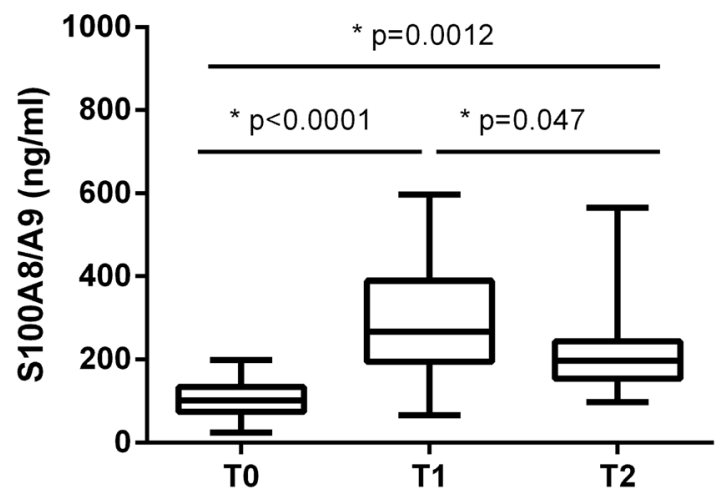

Fig. $2 \mathrm{MPO}(\mathrm{A})$ and S100A8/A9 (B) were increased in the sera of patients undergoing liver transplantation. As compared with T0 (before graft), both MPO and S100A8/A9 levels were increased remarkably at T1 (24 hours after graft), and showed a decreasing trend at T2 (within 72 hours after graft) but were still significantly higher than T0. Variables were expressed as median (interquartile range).

\subsection{Extracellular histones induced cellular damage and promoted cytokine production ex vivo}

We next asked whether the elevated extracellular histones after graft have a direct impact on cellular damage and systemic inflammation, which may further contribute to the occurrence of PGD or other adverse outcomes. We collected patients' sera at 24 hour post-transplantation which contained high levels of extracellular histones (median value, $26.2 \mu \mathrm{g} \mathrm{ml}^{-1}$ ) and incubated overnight with human L02 hepatocytes and human monocytes U937 cells, respectively. As compared with the sera obtained before graft (median value, $5.52 \mu \mathrm{g} \mathrm{ml}^{-1}$ ), patients' sera after graft had obviously damaging effects on L02 cells, as evidenced by decreased cell integrity (Fig. 4). Likewise, overnight culture of human U937 monocytes with patients' sera after graft led to a drastic increase in histone-associated cytokines (IFN- $\gamma$, IL-1 $\beta$, IL-6, IL-10, IL-18, TNF- $\alpha$ ) in the supernatants of cultured cells (Fig. 5).

Many studies have shown that therapeutic modulation of extracellular histones by anti-histone $\mathrm{H} 4$ antibody, activated protein C (APC) or heparin was highly protective in various inflammatory states..$^{14,26,27}$ In this study, we investigated whether heparin, which can bind histones, can offer protection for this condition. ${ }^{26}$ Anti-histone $\mathrm{H} 4$ antibody was also included. We observed that administration of heparin or anti-histone antibody equally inhibited the patients' sera-induced cell death or activation of U937 cells, which was evidenced by improved cell viability and decreased inflammatory cytokine levels (Fig. 4 and 5). These results thereby suggest a direct relationship between extracellular histones and cellular damage and systemic inflammation, and provide a possible therapeutic target in clinical practice.

\section{Discussion}

Liver transplantation (LT) is considered the best life-saving therapy for patients with end-stage liver disease. In recent decades, the development and broad administration of immunosuppressive drugs have contributed to increased graft

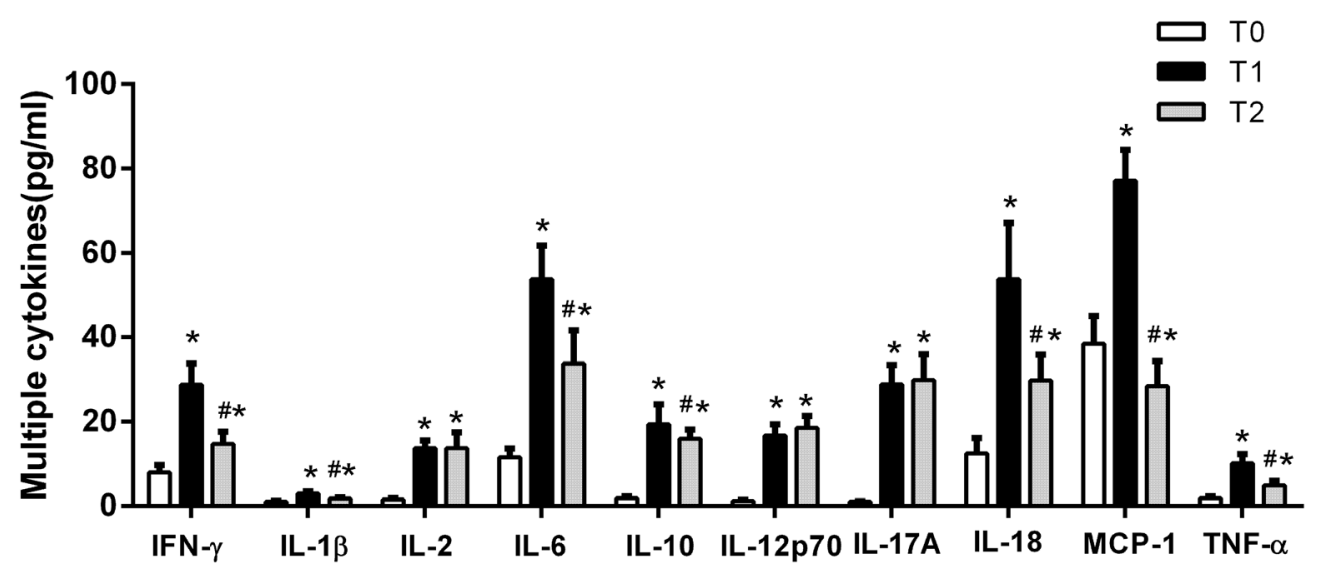

Fig. 3 Detection of systemic inflammation in the sera of patients undergoing liver transplantation. Multiplex immunoassay for a panel of multiple cytokines was performed. Only 10 cytokines with significant differences ( $* p<0.01$ in contrast to T0) were shown. \#p<0.01 denotes a significant difference between T1 and T2. Variables were expressed as median (interquartile range). 
Table 2 Correlation of extracellular histones with various variables in liver transplantation patients ${ }^{a}$

\begin{tabular}{lll}
\hline & $r$ & $p$ \\
\hline MPO & 0.5262 & 0.003 \\
S100A8/9 & 0.4738 & 0.015 \\
IFN- $\gamma$ & 0.5723 & 0.007 \\
IL-1 $\beta$ & 0.4047 & 0.037 \\
IL-6 & 0.6527 & 0.001 \\
IL-10 & 0.5894 & 0.001 \\
IL-18 & 0.6017 & 0.001 \\
TNF- $\alpha$ & 0.4179 & 0.037 \\
${ }^{a} p<0.05$ was considered to be statistically significant. &
\end{tabular}

survival rates. ${ }^{2,3}$ However, PGD still presents as one of most common complications after liver transplantation, which can affect the long-term postoperative outcomes. ${ }^{4}$ There were many risk factors for PGD. ${ }^{1}$ Of these, I/R injury is universally recognized as a major cause for LT-related PGD, further leading to higher episodes of both acute and chronic rejection. ${ }^{6,8}$ Emerging evidence suggests that extracellular histones are key mediators of I/R injury and targeting histones may attenuate I/ R-related cell and tissue damage. ${ }^{11,25}$ In this study, we sought to investigate whether extracellular histones are clinically involved with the occurrence of PGD in human liver transplantation.

Histones, which are highly conserved, positively charged proteins that include 5 main types (linker histone $\mathrm{H} 1$ and core

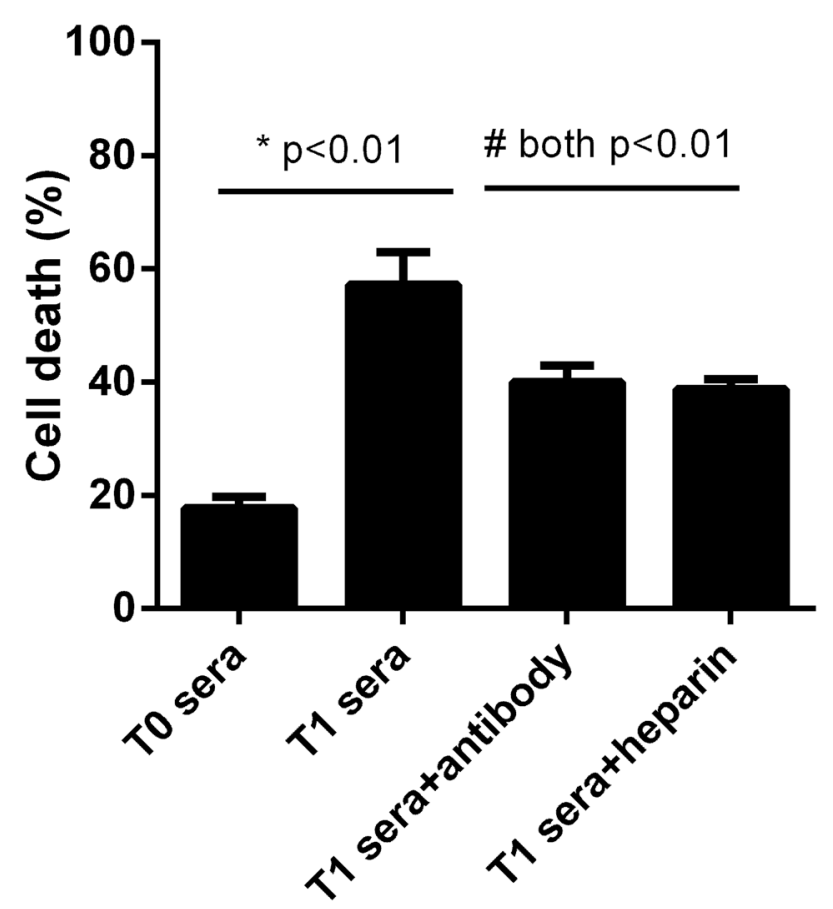

Fig. 4 Liver transplantation patients' sera induced human liver cell damage. It showed that the patients' sera at T1 that contained high levels of extracellular histones induced significant human liver cell damage after overnight incubation as compared with the sera collected at T0, whereas addition of anti-histone $\mathrm{H} 4$ antibody or heparin remarkably improved cell integrity. Variables were expressed as mean d standard deviation (SD). histones $\mathrm{H} 2 \mathrm{~A}, \mathrm{H} 2 \mathrm{~B}, \mathrm{H} 3$, and $\mathrm{H} 4$ ), have been traditionally investigated in the context of regulating nuclear architecture. ${ }^{11,25}$ However, under conditions of cellular stress or injury such as trauma, ischemic or chemical injury, or infection, histones can be released from both damaged cells and activated immune cells such as neutrophils into the extracellular space and/or blood. ${ }^{9,10,25,28}$ Extracellular histones are discovered to have the ability to cause endothelial cytotoxicity, platelet activation and aggregation, and act as danger/damage-associated molecular patterns (DAMPs) to initiate and propagate the inflammatory response, which may eventually lead to life threatening organ and tissue damages. ${ }^{29-31}$

The pathological role for extracellular histones in various disease processes has been investigated. However, little is known about clinical relevance of extracellular histones with PGD in human liver transplantation. Here we measured extracellular histone levels in patients undergoing LT. We found that extracellular histones were increased immediately after LT and peaked at around 24 hours with high levels still detectable at 72 hours post-transplantation. The elevated extracellular histones are likely due to $\mathrm{I} / \mathrm{R}$ injury occurring during transplantation. ${ }^{32}$ Many studies have confirmed that I/R injury of the liver, brain, and kidney caused an elevation of extracellular histones in the circulation..$^{13,33,34}$ More importantly, we observed that compared with patients without PGD, patients with PGD had significantly higher levels of extracellular histones after transplantation, thereby suggesting the likelihood that extracellular histones are an important cause for PGD. In addition, we detected a remarkable immune cell activation and a significant inflammatory response during the course of transplantation. There was a clear correlation between extracellular histones and immune cell activation and systemic inflammation, which was consistent with previous reports that extracellular histones can promote systemic inflammation through activation of immune cells particularly neutrophils. ${ }^{19,35,36}$

Based on these observations, we concluded that liver transplantation-related $\mathrm{I} / \mathrm{R}$ injury causes death of tissues and cells, and leads to the release of histones from these dying cells into extracellular space such as the circulation. Large quantities of extracellular histones are capable of inducing direct cytotoxicity, or promoting platelet aggregation and coagulation disturbance, as well as act as DAMP molecules to amplify systemic inflammation by stimulating immune cells (e.g. neutrophils) to produce multiple cytokines, ${ }^{9}$ which eventually contribute to PGD or other complications after graft.

To further validate the above findings, we incubated the patients' post-transplantation sera that contained high levels of extracellular histones with human liver cells and monocytes, and observed that the post-transplantation sera could induce massive cultured liver cell death as well as cause a significant production in histone-related cytokines in cultured monocytes. To test whether these effects were majorly due to extracellular histones, we used a specific anti-histone $\mathrm{H} 4$ antibody and found anti-histone treatment could prevent these adverse effects, thereby confirming that the elevated extracellular histones are a major mechanism for organ injury such as PGD after transplantation. These findings also suggest that blockade of 

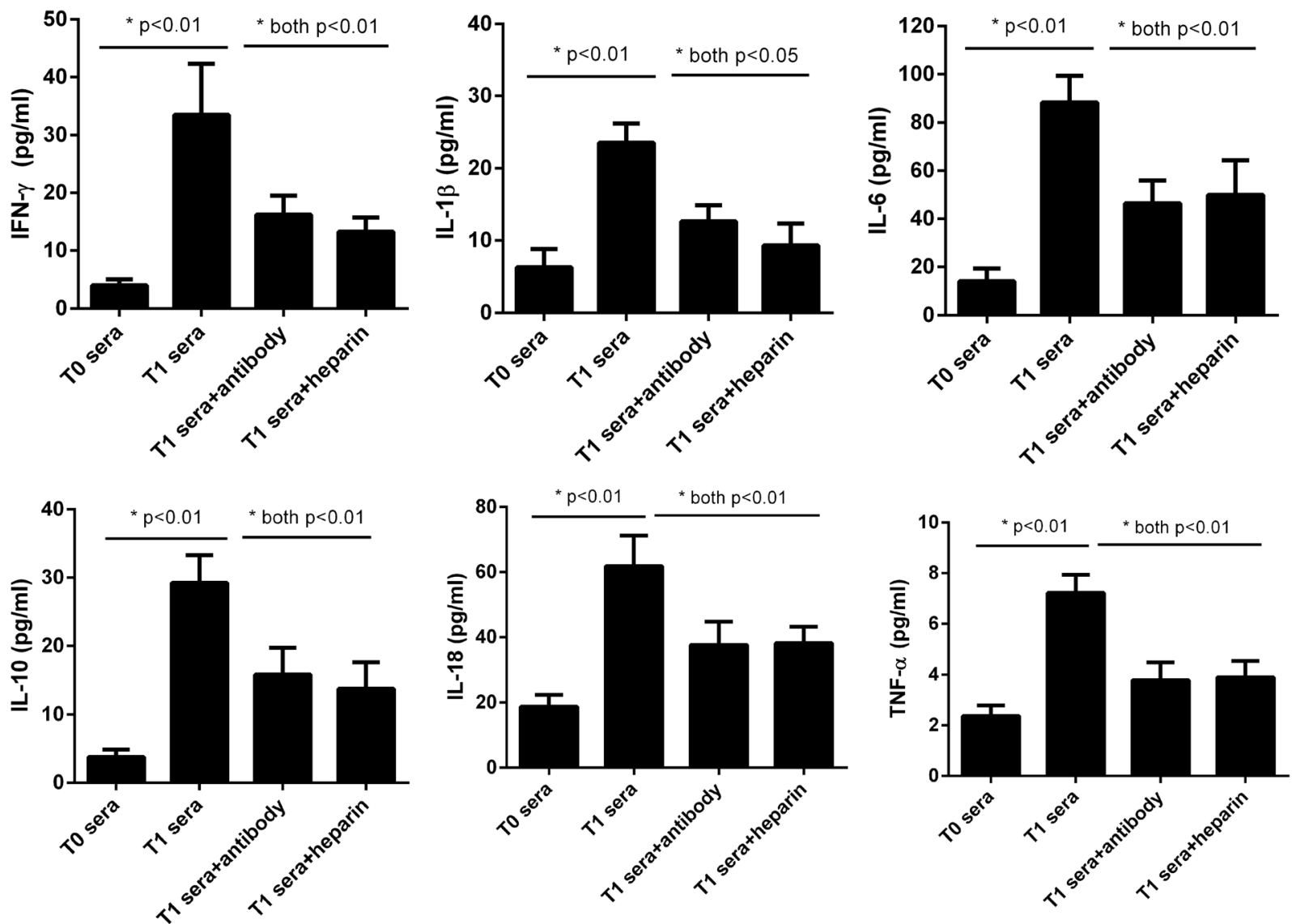

Fig. 5 Liver transplantation patients' sera stimulated human monocytic cells. It showed that 6 histone-related cytokines were all notably increased in the supernatant of human monocytic U937 cells treated by liver transplantation patients' sera collected at T1 (24 hours after graft), whereas addition of anti-histone $\mathrm{H} 4$ antibody or heparin could decrease these cytokine levels (all $p<0.05$ ). Variables were expressed as mean . Ostandard deviation (SD).

extracellular histones may be a promising therapeutic strategy for PGD or other complications. We then assessed the interventional effects of other histone blocking agent such as heparin in this study. It has been reported that heparin, which can bind histones, showed potent protective effects in histonemediated inflammatory injuries including sepsis, acute liver injury, and acute respiratory distress syndrome (ARDS). ${ }^{\mathbf{1 1 , 1 4 , 2 6}}$ Here, we observed that heparin had a similarly cytoprotective effect as anti-histone antibody in decreasing histone-induced cytotoxicity or cytokine production for this condition, which may provide a potential approach for clinical management of PGD or other complications after liver transplantation.

In summary, our results provide a credible explanation for the mechanisms underlying PGD or other complications after graft. Determination of extracellular histones may become a promising tool for assessing prognosis in human liver transplantation. In addition, histone-targeted strategies may be potentially therapeutic in clinical practice. More histone blocking agents deserve exploration in the future.

\section{Conflicts of interest}

The authors declare that they have no conflict of interest.

\section{Acknowledgements}

This work was supported by grants from National Natural Science Foundation of China (81473500, to Xihui Li), Beijing, P. R. China; and by Beijing Natural Science Foundation (7172079, to Tao Wen).

\section{References}

1 X. B. Chen and M. Q. Xu, Primary graft dysfunction after liver transplantation, Hepatobiliary Pancreatic Dis. Int., 2014, 13(2), 125-137.

2 R. F. Meirelles Junior, P. Salvalaggio, M. B. Rezende, A. S. Evangelista, B. D. Guardia, C. E. Matielo, et al., Liver transplantation: history, outcomes and perspectives, Einstein, 2015, 13(1), 149-152.

3 A. Zarrinpar and R. W. Busuttil, Liver transplantation: past, present and future, Nat. Rev. Gastroenterol. Hepatol., 2013, 10(7), 434-440.

4 D. D. Lee, K. P. Croome, J. A. Shalev, K. R. Musto, M. Sharma, A. P. Keaveny, et al., Early allograft dysfunction after liver transplantation: an intermediate outcome measure for targeted improvements, Ann. Hepatol., 2016, 15(1), 53-60. 
5 N. Golse, N. Guglielmo, A. El Metni, F. Frosio, C. Cosse, S. Naili, et al., Arterial Lactate Concentration at the End of Liver Transplantation is an Early Predictor of Primary Graft Dysfunction, Ann. Surg., 2018, DOI: 10.1097/ SLA.0000000000002726.

6 H. Bruns, J. Heil, D. Schultze, M. Al Saeedi and P. Schemmer, Early markers of reperfusion injury after liver transplantation: association with primary dysfunction, Hepatobiliary Pancreatic Dis. Int., 2015, 14(3), 246-252.

7 M. Z. Molnar, K. Joglekar, Y. Jiang, G. Cholankeril, M. K. M. Abdul, S. Kedia, et al., Association of PreTransplant Renal Function with Liver Graft and Patient Survival after Liver Transplantation in Patients with Nonalcoholic Steatohepatitis, Liver Transpl., 2019, 25(3), 399-410.

8 J. M. Ali, S. E. Davies, R. J. Brais, L. V. Randle, J. R. Klinck, M. E. Allison, et al., Analysis of ischemia/reperfusion injury in time-zero biopsies predicts liver allograft outcomes, Liver Transpl., 2015, 21(4), 487-499.

9 E. Silk, H. Zhao, H. Weng and D. Ma, The role of extracellular histone in organ injury, Cell Death Dis., 2017, 8(5), e2812.

10 R. Allam, S. V. Kumar, M. N. Darisipudi and H. J. Anders, Extracellular histones in tissue injury and inflammation, $J$. Mol. Med., 2014, 92(5), 465-472.

11 P. Szatmary, W. Huang, D. Criddle, A. Tepikin and R. Sutton, Biology, role and therapeutic potential of circulating histones in acute inflammatory disorders, J. Cell. Mol. Med., 2018, 22(10), 4617-4629.

12 C. Kawai, H. Kotani, M. Miyao, T. Ishida, L. Jemail, H. Abiru, et al., Circulating Extracellular Histones Are Clinically Relevant Mediators of Multiple Organ Injury, Am. J. Pathol., 2016, 186(4), 829-843.

13 R. Allam, C. R. Scherbaum, M. N. Darisipudi, S. R. Mulay, H. Hagele, J. Lichtnekert, et al., Histones from dying renal cells aggravate kidney injury via TLR2 and TLR4, J. Am. Soc. Nephrol., 2012, 23(8), 1375-1388.

14 E. Lefrancais and M. R. Looney, Neutralizing Extracellular Histones in Acute Respiratory Distress Syndrome. A New Role for an Endogenous Pathway, Am. J. Respir. Crit. Care Med., 2017, 196(2), 122-124.

15 S. T. Abrams, N. Zhang, C. Dart, S. S. Wang, J. Thachil, Y. Guan, et al., Human CRP defends against the toxicity of circulating histones, J. Immunol., 2013, 191(5), 2495-2502.

16 J. Xu, X. Zhang, M. Monestier, N. L. Esmon and C. T. Esmon, Extracellular histones are mediators of death through TLR2 and TLR4 in mouse fatal liver injury, J. Immunol., 2011, 187(5), 2626-2631.

17 H. Huang, H. W. Chen, J. Evankovich, W. Yan, B. R. Rosborough, G. W. Nace, et al., Histones activate the NLRP3 inflammasome in Kupffer cells during sterile inflammatory liver injury, J. Immunol., 2013, 191(5), 26652679.

18 V. Perilli, P. Aceto, C. Modesti, P. Ciocchetti, T. Sacco, F. Vitale, et al., Low values of left ventricular ejection time in the post-anhepatic phase may be associated with occurrence of primary graft dysfunction after orthotopic liver transplantation: results of a single-centre case-control study, Eur. Rev. Med. Pharmacol. Sci., 2012, 16(10), 14331440.

19 M. Bosmann, J. J. Grailer, R. Ruemmler, N. F. Russkamp, F. S. Zetoune, J. V. Sarma, et al., Extracellular histones are essential effectors of C5aR- and C5L2-mediated tissue damage and inflammation in acute lung injury, FASEB J., 2013, $27(12)$, 5010-5021.

20 O. E. Sorensen and N. Borregaard, Neutrophil extracellular traps - the dark side of neutrophils, J. Clin. Invest., 2016, 126(5), 1612-1620.

21 O. Elaskalani, N. B. Abdol Razak and P. Metharom, Neutrophil extracellular traps induce aggregation of washed human platelets independently of extracellular DNA and histones, Cell Commun. Signaling, 2018, 16(1), 24.

22 S. V. Kumar, O. P. Kulkarni, S. R. Mulay, M. N. Darisipudi, S. Romoli, D. Thomasova, et al., Neutrophil Extracellular Trap-Related Extracellular Histones Cause Vascular Necrosis in Severe GN, J. Am. Soc. Nephrol., 2015, 26(10), 2399-2413.

23 J. Westman, P. Papareddy, M. W. Dahlgren, B. Chakrakodi, A. Norrby-Teglund, E. Smeds, et al., Extracellular Histones Induce Chemokine Production in Whole Blood Ex Vivo and Leukocyte Recruitment In Vivo, PLoS Pathog., 2015, 11(12), e1005319.

24 R. Allam, M. N. Darisipudi, J. Tschopp and H. J. Anders, Histones trigger sterile inflammation by activating the NLRP3 inflammasome, Eur. J. Immunol., 2013, 43(12), 3336-3342.

25 M. Hoeksema, M. van Eijk, H. P. Haagsman and K. L. Hartshorn, Histones as mediators of host defense, inflammation and thrombosis, Future Microbiol., 2016, 11(3), 441-453.

26 K. C. Wildhagen, P. Garcia de Frutos, C. P. Reutelingsperger, R. Schrijver, C. Areste, A. Ortega-Gomez, et al., Nonanticoagulant heparin prevents histone-mediated cytotoxicity in vitro and improves survival in sepsis, Blood, 2014, 123(7), 1098-1101.

27 K. Daigo, Y. Takamatsu and T. Hamakubo, The Protective Effect against Extracellular Histones Afforded by LongPentraxin PTX3 as a Regulator of NETs, Front. Immunol., 2016, 7, 344.

$28 \mathrm{~W}$. $\mathrm{Xu}$ and Y. Song, Biomarkers for patients with trauma associated acute respiratory distress syndrome, Mil. Med. Res., 2017, 4, 25.

29 J. Xu, X. Zhang, R. Pelayo, M. Monestier, C. T. Ammollo, F. Semeraro, et al., Extracellular histones are major mediators of death in sepsis, Nat. Med., 2009, 15(11), 1318-1321.

30 Y. Zhang, Z. Wen, L. Guan, P. Jiang, T. Gu, J. Zhao, et al., Extracellular histones play an inflammatory role in acid aspiration-induced acute respiratory distress syndrome, Anesthesiology, 2015, 122(1), 127-139.

31 X. Lv, T. Wen, J. Song, D. Xie, L. Wu, X. Jiang, et al., Extracellular histones are clinically relevant mediators in the pathogenesis of acute respiratory distress syndrome, Respir. Res., 2017, 18(1), 165. 
32 T. Li, W. Lee, H. Hara, C. Long, M. Ezzelarab, D. Ayares, et al., An Investigation of Extracellular Histones in Pig-To-Baboon Organ Xenotransplantation, Transplantation, 2017, 101(10), 2330-2339.

33 H. Huang, J. Evankovich, W. Yan, G. Nace, L. Zhang, M. Ross, et al., Endogenous histones function as alarmins in sterile inflammatory liver injury through Toll-like receptor 9 in mice, Hepatology, 2011, 54(3), 999-1008.

34 S. F. De Meyer, G. L. Suidan, T. A. Fuchs, M. Monestier and D. D. Wagner, Extracellular chromatin is an important mediator of ischemic stroke in mice, Arterioscler., Thromb., Vasc. Biol., 2012, 32(8), 1884-1891.

35 S. T. Abrams, N. Zhang, J. Manson, T. Liu, C. Dart, F. Baluwa, et al., Circulating histones are mediators of traumaassociated lung injury, Am. J. Respir. Crit. Care Med., 2013, 187(2), 160-169.

36 D. S. Pisetsky, Immune activation by histones: plusses and minuses in inflammation, Eur. J. Immunol., 2013, 43(12), 3163-3166. 